\title{
Leptospirosis in Pregnancy: A Rare Condition Mimicking HELLP Syndrome
}

\author{
Carmen Tonga, ${ }^{\mathrm{a}}$, Manisha Mathur ${ }^{\mathrm{a}}$
}

\begin{abstract}
We report a case of leptospirosis in pregnancy. A 37-year-old woman of para 1, normal vaginal delivery (NVD) presented at 33 weeks with 3 days of jaundice and diarrhea and no history of fever, abdominal pain, itch or pale stools. Her pregnancy was uneventful except for gestational diabetes. On examination she had jaundice otherwise unremarkable. Investigations revealed proteinuria, hemolysis, low platelets, raised transaminases and bilirubin. Working diagnosis was of atypical hemolysis, elevated liver enzymes, low platelets (HELLP) syndrome owing to absence of hypertension. She progressed into labour 1 day later after preterm premature rupture of membranes (PPROM), had thick meconium and pathological cardiotocography (CTG). She delivered a baby weighing 1,736 $\mathrm{g}$ and normal Apgar scores. Arterial cord $\mathrm{pH}$ was 7.275 , lactate 3.04 , base excess of -8.8 and venous $\mathrm{pH}$ was 7.316 , lactate 2.93, BE -10.6. Leptospirosis EIA IgM antibody was positive and placenta histology showed focus increased perivillous fibrinoid deposits with acute intervillositis and meconium staining. Baby showed no signs of congenital leptospirosis and was discharged on day 15 of life. Patient's symptoms resolved within 2 weeks and her biochemistry normalized within 1 month. Owning to unusual presentation and rarity, leptospirosis in pregnancy is often misdiagnosed and underreported. It mimics HELLP syndrome, viral hepatitis, obstetric cholestasis and acute fatty liver of pregnancy. It has severe implications of early miscarriages, stillbirths and neonatal leptospirosis requiring high index of suspicion, need for increased awareness, early diagnosis and treatment.
\end{abstract}

Keywords: Leptospirosis; Pregnancy; HELLP

\section{Introduction}

Leptospirosis is an endemic disease in tropical countries and one of the most widespread zoonotic diseases in the world. Owing to unusual presentation and rarity, leptospirosis in pregnancy is often misdiagnosed and under-reported. It mim-

Manuscript submitted May 5, 2018 KK women and Children Hospital, Singapore 229899, Singapore

${ }^{b}$ Corresponding Author: Carmen Tong, KK Women and Children Hospital, 100 Bukit Timah Road, Singapore 229899, Singapore.

Email: Carmen.tong@mohh.com.sg

doi: https://doi.org/10.14740/jmc3073w ics HELLP syndrome, viral hepatitis, obstetric cholestasis and acute fatty liver of pregnancy. It has severe implications of early miscarriages, stillbirths and neonatal leptospirosis, and requires high index of suspicion, necessitates increased awareness, early diagnosis and treatment.

We report a case of leptospirosis in a 37-year-old at 33 weeks of pregnancy. She initially presented with jaundice, diarrhea and recent travel history to Vietnam. Initial workup pointed towards a diagnosis of atypical hemolysis, elevated liver enzymes, low platelets (HELLP) syndrome in view of a hemolysis picture with proteinuria but subsequent leptospirosis $\lg M$ was positive. She was treated with P.O. doxycycline and had a preterm delivery at 33 weeks. We reviewed the literature and discussed the possible differentials that could overlap in presentation with leptospirosis. The authors believe this could be of educational interest to obstetricians as it is a rare infectious disease.

\section{Case Report}

A 37-year-old multip with the history of one full-term normal vaginal delivery presented to the labour ward with non bilious, non bloody diarrhea for 3 days at $33+2$ weeks. This was associated with nausea, abdominal pain and tea-coloured urine. She has no past medical or surgical history, no past family history of liver disease and did not use any drugs or traditional supplements. She had a travel history to Ho Chi Minh City 3 months prior to admission but no social history of smoking or drinking. Her current pregnancy was uncomplicated except for gestational diabetes mellitus on diet control.

On examination, there was no confusion or lethargy. Patient looked non-toxic. She remained afebrile, blood pressure was normal throughout admission. There was sclera icterus and she was jaundiced, otherwise no stigmata of chronic liver disease. There was no hepatosplenomegaly or right hypochondriac tenderness. Hepatic flap was negative. Speculum examination showed that the cervical os was closed.

Initial blood results showed that hemoglobin was stable at $10 \mathrm{~g} / \mathrm{dL}$, platelets downtrending from 254 to $195 \times 10^{9} / \mathrm{L}$, total white blood cells increasing from 12.3 to $27.2 \times 10^{9} / \mathrm{L}$ with deranged APTT/PT. Liver function tests (LFT) shows aspartate transaminase (AST) $155 \mathrm{U} / \mathrm{L}$, alanine transaminase (ALT) 153 $\mathrm{U} / \mathrm{L}$, gamma-glutamyl transferase (GGT) $238 \mathrm{U} / \mathrm{L}$ with raised total bilirubin $166 \mu \mathrm{mol} / \mathrm{L}$ and direct bilirubin $123 \mu \mathrm{mol} / \mathrm{L}$ as well as acute kidney injury with a creatinine of 117.

Serological test for infection was performed which con- 
firmed that hepatitis A, B, C, E, CMV, EBV and HIV were all negative. Blood and urine cultures were also negative. Autoimmune tests ANA and anti liver antibody were negative. Ultrasound of the hepatobiliary system was normal, showing no hepatic lesion or intrahepatic biliary ductal dilatation. The common bile duct was not dilated. Doppler ultrasound (US) of liver was done and there was no evidence of hepatic venous thrombosis and the portal vein was patent.

The working diagnosis was of atypical HELLP syndrome (absence of hypertension) in view of low platelets and haptoglobin, raised uric acid, $\mathrm{LDH}$ and liver transaminases with proteinuria.

She was transferred to labour ward 2 days later for unprovoked decelerations seen on cardiotocography. A few hours later, she had spontaneous rupture of membranes which showed thick meconium stained liquor. She delivered via normal vaginal delivery at 33+4 weeks, Apgar 9.9, birth weight 1,736 g. Baby's arterial cord blood showed $\mathrm{pH} 7.275$, lactate 3.04, BE -8.8 , venous cord blood showed $\mathrm{pH} 7.316$, lactate $2.93, \mathrm{BE}$ -10.6 . Placenta for histology showed focal increased perivillous fibrinoid associated with acute intervillositis, meconium staining of membranes, no leptospirosis detected.

She was given 1 pint of fresh frozen plasma in view of deranged coagulopathy and discharged against advice on post delivery day. She was readmitted on post delivery day 8 as leptospirosis enzyme-linked immunosorbent assay (ELISA) immunoglobulin $\mathrm{M}(\operatorname{Ig} M)$ +ve and she was still having intermittent diarrhea.

She was referred to the infectious disease team and started on oral doxycycline and advised to withhold breastfeeding for 2 weeks. Baby was initially covered with IV ampicillin and gentamicin for presumptive Listeria. In view of maternal diarrhea, she stopped consuming breast milk on day 8 of life. Her liver function tests and renal panel was normal. She showed no signs of congenital leptospirosis and was discharged well on day 15 of life at $1,800 \mathrm{~g}$.

\section{Discussion}

Rare cases of leptospirosis in pregnancy are reported in literature. Human infections can occur by direct contact with urine or tissue of an infected animal, especially rodents or indirectly through contaminated water, soil, or vegetation [1]. Leptospirosis ranges in severity from a mild illness suggesting a viral infection to a multisystemic syndrome. The clinical presentation is non-specific like fever, headache, chills, abdominal pain, diarrhea, anorexia, vomiting, lymphadenopathy, rash and hepatosplenomegaly [2]. Icteric leptospirosis, or Weil's syndrome, characterized by liver, kidney, and vascular dysfunction, occurs in $5-10 \%$ of cases and has a fatality rate up to $20-40 \%$ [2].

Laboratory studies used to screen for the diagnosis of leptospirosis include IgM ELISA or IgM/immunoglobulin G (IgG) ELISA or real-time DNA polymerase chain reaction (PCR) of blood, urine, and cerebrospinal fluid (CSF). Laboratory studies used to confirm the diagnosis of leptospirosis include a single titer $\geq 1: 200$ or 4-fold rise in microscopic agglutination testing (MAT) titre drawn between the first and fourth week of illness or culture of leptospires from body fluids or tissue [3-4].

Treatment with antibiotics like penicillin $\mathrm{G}$, streptomycin, doxycycline, chloramphenicol, and erythromycin have been effective in treating these patients [4]. WHO reported that leptospirosis during pregnancy may lead to abortion, fetal death, stillbirth or congenital leptospirosis in women depending of the period of pregnancy [4].

In terms of fetal implications, Yechiel et al [5] reviewed 15 previously reported cases of leptospirosis in pregnancy, which showed abortion in eight women, healthy babies in two, and four babies with signs of active leptospirosis. Puliyath et al [2] reported that leptospirosis is transmitted through breast milk and can result in neonatal leptospirosis. Therefore, mothers with suspected postpartum leptospirosis should immediately stop breastfeeding and the infant should be tested for Leptospira infection.

Hepatobiliary diseases during pregnancy can be divided into pregnancy related liver disease such as acute fatty liver of pregnancy, HELLP, obstetric cholestasis or liver diseases unrelated to pregnancy like gallstones, hepatitis or other infections. In the third trimester, abdominal pain associated with jaundice, hemolysis, raised transaminases and coagulations abnormalities, likely differentials include HELLP syndrome and AFLP [6]. Both disorders show similar clinical and laboratory presentation and accurate diagnosis will be challenging [7]. Hypoglycemia will be present in AFLP and ideally, a liver biopsy should be performed to rule out AFLP. In view of patient's travel and hygiene history, it is highly suggestive of an infection and leptospirosis must be ruled out.

In conclusion, leptospirosis is uncommon and difficult to diagnose due to its rare and non specific clinical presentation. It is therefore important to have a high index of suspicion, render early diagnosis and treatment and to avoid severe implications of early miscarriages, stillbirths and neonatal leptospirosis. Leptospirosis is an uncommon infection that mimics other conditions like HELLP or acute fatty liver in pregnancy, high index of suspicion is needed for early diagnosis of this condition. Obstetrician/gynecologist needs to be aware of fetal complications of leptospirosis including early miscarriages, stillbirths, preterm labour and neonatal leptospirosis. Multidisciplinary care consisting of nephrologists, hematologists, gastroenterologists, obstetricians and the infectious disease team are needed in the management of leptospirosis in particular patients with severe leptospirosis or Weil's syndrome.

\section{Conflict of Interest}

No conflict of interest in this study and no grant involved.

\section{References}

1. Hicham S, Ihsane M, Abderahim el B, Brahim B, Labib $\mathrm{S}$, Mustapha H, Mohamed K, et al. Multivisceral organ failure related to leptospirosis in pregnant patient. Indian J Crit Care Med. 2013;17(1):43-45.

2. Puliyath G, Singh S. Leptospirosis in pregnancy. Eur J 
Clin Microbiol Infect Dis. 2012;31(10):2491-2496.

3. Cardenas-Marrufo MF, Vado-Solis I, Perez-Osorio C, Peniche-Lara G, Segura-Correa J. A cross sectional study of leptospirosis and fetal death in Yucatan, Mexico. Colomb Med (Cali). 2016;47(1):11-14.

4. World Health Organization. Human leptospirosis: guidance for diagnosis, surveillance and control. Geneva: World Health Organization. 2003.

5. Shaked Y, Shpilberg O, Samra D, Samra Y. Leptospirosis in pregnancy and its effect on the fetus: case report and review. Clin Infect Dis. 1993;17(2):241-243.

6. Lata I. Hepatobiliary diseases during pregnancy and their management: An update. Int J Crit Illn Inj Sci. 2013;3(3):175-182.

7. Gaspari R, Annetta MG, Cavaliere F, Pallavicini F, Grillo $\mathrm{R}$, Conti G, Antonelli M, et al. Unusual presentation of leptospirosis in the late stage of pregnancy. Minerva Anestesiol. 2007;73(7-8):429-432. 